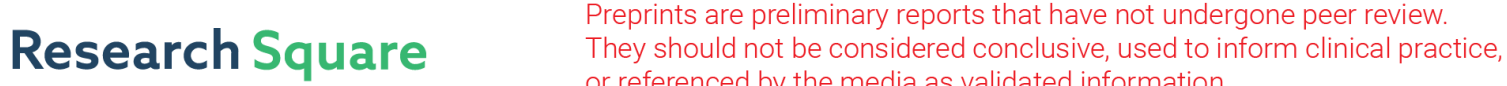 or referenced by the media as validated information. \\ Perturbative norm for associated spreading of diseases
}

Deep Bhattacharjee ( $\square$ itsdeep@live.com )

AATWRI-Research Directorate EGSPL https://orcid.org/0000-0003-0466-750X

Aruna Harikant

IIT Mandi https://orcid.org/0000-0001-9520-6404

\section{Research Article}

Keywords: Instigation Factors, Immunization, Spanning , Strain , Infection Cycle, Waves , Co-morbidity , Cure Parameter

Posted Date: December 1st, 2020

DOI: https://doi.org/10.21203/rs.3.rs-117165/v1

License: (c) (i) This work is licensed under a Creative Commons Attribution 4.0 International License. Read Full License 


\title{
Perturbative norm for associated spreading of diseases
}

\author{
Deep Bhattacharjee ${ }^{1}$, Aruna Harikant ${ }^{2}$ \\ ${ }^{* 1}$ University of Calcutta, 87, 1, College Street, College Square, West Bengal, Kolkata - 700073 \\ ${ }^{1}$ Researcher at AATWRI Aerospace and Defense, Electro-Gravitation Simulation and Propulsion Laboratory, Orissa \\ ${ }^{2}$ Indian Institute of Technology Mandi, Parashar Road, Tehsil Sadar, Kamand, Himachal Pradesh 175005
}

\begin{abstract}
The mathematical modelling of a pandemic with prevention, mutation and infection parameters to justify the natural properties of the diseases with a view in controlling it. Strength of the virus strain, population density gradient, cyclic healthcare potentials (with vaccination and cure) along with 2 WAVES (generalized upto ' $n$ ' waves with dual and anti-dual factors) interpreted over periodic cycles of odd and even permutations to illuminate the necessary conditions for natural and artificial immunization thereby emanating the potential for cures over $(-3, \pm 1,+3)$ instigation factors as interpreted over a Bell curve.
\end{abstract}

Received -

Keywords: Instigation Factors - Immunization - Spanning - Strain - Infection CycleWaves - Co-morbidity - Cure Parameter.

Introduction: The diseases in the human population are extensively studied by mathematical modeling to justify the natural properties of the diseases in a view to control it. The diseases present an urgent threat concerned with global health and its effects are expected to get worsen in the underdeveloped countries where the health system is weak and fragile. In this paper, the phenomena of the spread of disease have been mathematically analyzed by the matrix method keeping into account all the factors such as mutation, strains, prevention, protection, and spread factors. In the methodology section, the spreading of diseases is assumed as growth of wave cycles which approaches with mass immunization resulting from the mass transmission of diseases and vaccination which will produce antibodies and then the origin of new cycles begins which will diminish as recovery rate gets dominant over infected rate with almost no death.

In the end, it is concluded by relating the span factor in equation (1) to the viral factor in equation (2), to the strength of strain factor in equation (3), a body has been build up to cope with the Dual and Anti-Dual factors along with the population density gradient and the $(+3,+-1,-3)$ instigation factors. This would be another new insight into the epidemiological mathematical model. The appendix is also included in this paper which describes the plots of diseases such as cancer, HIV as co-morbid to recent SARS Covid-19 along with original plots of the SARS Covid-19 Parameters etcetera.

Methodology: To develop a theory from the base requires certain mathematical parameterizations', although there existed previous theories [1-8], with one of the most prominent SIR models [9-11], and the recent Covid-19 [12,13] but still, we believed that this will be a more concrete and profound modeling with a sole view to check the spreading of the disease, keeping into account all the related factors like mutation, strains, prevention, protection and spread factors, a new hope is always there to create something practical, new and useful for the benefit of humanity.

The periodicity of the pandemic would be the main and foremost parameter denoting the periodicity as $\Delta P$ and pandemic as $\oplus$ in contemplating $\Delta P=\frac{F_{0}-L}{S p a n}$ where $F_{0}$ is the 'first' moment in human civilization as recorded by humans the start of a pandemic, $L$ being the latest moment of the last as relative to present and Span is the gap in years between two successive events (from first as recorded to the next one, to the last successively), as correlated by $F_{0}-F_{1}-F_{2}-F_{3}-F_{4} \ldots \ldots \ldots L$, however it is plausible to ignore the middle factors $F_{1,2,3,4, \ldots . . .}$ as we have taken the Span, thereby concluding to the master equation as,

$$
\bigoplus^{\Delta P}=\bigoplus^{\frac{F_{0}-L}{S p a n}} \quad \exists F_{0}, L, \text { Span } \in \mathbb{R}
$$

Correspondence to be addressed at itsdeep@live.com

${ }^{*}$ Former Student of University of Calcutta 
The viral factor however could be denoted by $\Pi^{\mathrm{D}-1}$ where $\mathrm{D}$ is the 'degree' and if $\mathrm{D} \cong 1$, then the virus will have no impact as the equation suffices $\Pi^{0}$ providing $\mathrm{D}>1$ when there is 'pandemic like virus' and $\mathrm{D}=1$ when the virus is not categorized as a pandemic. $\mathrm{D}$ can take the values $\gg 1$ if the virus causes a pandemic to last long such that $\mathrm{D}$ is dependent on a 'time factor' $T$ giving the equation,

$$
\Pi^{\mathrm{D}-1} \bar{\partial} T
$$

It is worthwhile to mention the strength of the virus $\varphi_{N}^{* 2}$ as an increasing magnitude from 1 to ...... depending on the strain evolution factor $\kappa^{-\bar{\partial} T}$. Here the strain evolution factor is an inverse 'time factor' provided the strain develops faster, if the strength $\varphi_{N}^{*^{2}}$ is stronger denoting a far catastrophe in a less time as the strength depends upon 2 factors of operation, where the probability $*^{2}=1$ keeping the strength viable through the values, $\varphi_{-K}^{*^{2}}, \ldots, \varphi_{0}^{*^{2}}, \varphi_{1}^{*^{2}}, \varphi_{2}^{*^{2}}, \varphi_{3}^{*^{2}}, \ldots \varphi_{+K}^{*^{2}}$ provided $+K \gg-K$ and $K, N \in \mathbb{R}$, thereby giving us the equation,

$$
\int_{\oplus^{F_{0}-L}}^{\pi \text { Span }} \varphi_{-K}^{*^{2}}, \ldots, \varphi_{0}^{*^{2}}, \varphi_{1}^{*^{2}}, \varphi_{2}^{*^{2}}, \varphi_{3}^{*^{2}}, \ldots \varphi_{+K}^{*^{2}}\left(\wedge^{-\bar{\partial} T}\right) \quad \pi: \bigoplus^{\frac{F_{0}-L}{S p a n}} \rightarrow \infty \exists \pi \text { is the mapping factor }
$$

The mutation factor $\omega^{ \pm}$is the 6 coordinate factors, such that, it develops a tree level structure to denote a mapping matrix, from the strong mutation $\omega^{+}$and weak mutation $\omega^{-}$where the 'weak mutation factor' $\omega^{-}$vanishes leading to the strong mutation factor $\omega^{+}$to take on the values of 4 kinds divided into 2 sections,

$$
\begin{array}{ll}
\text { Weak }: \omega^{-} \rightarrow \doteqdot & \text { Moderate }: \omega^{+} \rightarrow \fallingdotseq \\
\text { Strong }: \omega^{+} \rightarrow \risingdotseq & \text { Extreme }: \omega^{+} \rightarrow \risingdotseq+\fallingdotseq
\end{array}
$$

This provides the structures as $\omega^{-}$projects to $N I L$ whereas, $\omega^{+}$projects to $\doteqdot, \fallingdotseq, \risingdotseq, \risingdotseq+\fallingdotseq$ such that,

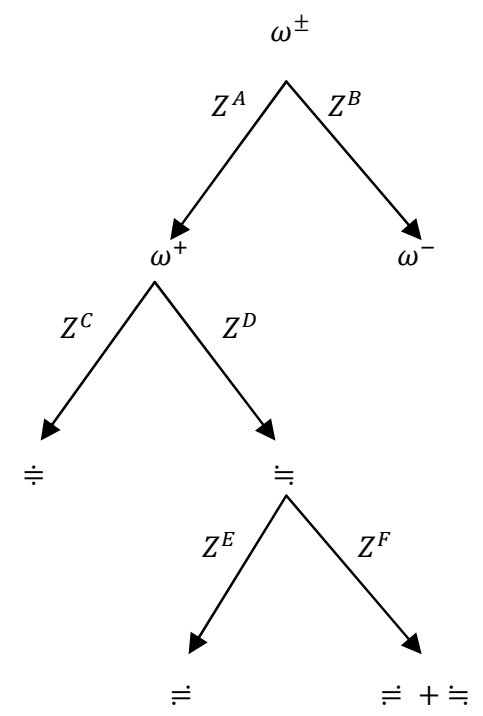

The corresponding 2 coordinates are non-null as $\left(Z^{A}, Z^{D}\right)=\left(\omega^{+}, \fallingdotseq\right)$ and null 4 coordinates are $\left(Z^{B}, Z^{C}, Z^{E}, Z^{F}\right)=\left(\omega^{-}, \doteqdot, \risingdotseq\right.$ ,$\risingdotseq+\doteqdot$. Apart from the null coordinates or the weak, a nice metric could be generated from this as Jnon-nullMN and JnullPQ as the following,

$$
J_{\text {non-null }}^{M N}=\left(\begin{array}{cccc}
\omega^{+} & 0 & 0 & 0 \\
0 & \fallingdotseq & 0 & 0 \\
0 & 0 & 0 & 0 \\
0 & 0 & 0 & 0
\end{array}\right) \quad J_{\text {non-null }}^{M N} \mathcal{L}_{M} \mathcal{L}_{N} \text { for the disease vector } \mathcal{L}
$$




$$
\begin{aligned}
& J_{\text {null }}^{P Q}=\left(\begin{array}{cccc}
\omega^{-} & 0 & 0 & 0 \\
0 & \doteqdot & 0 & 0 \\
0 & 0 & \risingdotseq & 0 \\
0 & 0 & 0 & \risingdotseq+\fallingdotseq
\end{array}\right) \quad \quad J_{\text {null }}^{P Q} \ell_{P} \ell_{Q} \text { for the disease vector } \ell \\
& J_{\text {non-null }}^{M N} \times J_{\text {null }}^{P Q}=\left(\begin{array}{cccc}
\omega^{+} & 0 & 0 & 0 \\
0 & \doteqdot & 0 & 0 \\
0 & 0 & 0 & 0 \\
0 & 0 & 0 & 0
\end{array}\right) \times\left(\begin{array}{cccc}
\omega^{-} & 0 & 0 & 0 \\
0 & \doteq & 0 & 0 \\
0 & 0 & \risingdotseq & 0 \\
0 & 0 & 0 & \risingdotseq+\fallingdotseq
\end{array}\right)=\left[\begin{array}{cccc}
\omega^{+} \times \omega^{-} & 0 & 0 & 0 \\
0 & \fallingdotseq \times \doteqdot & 0 & 0 \\
0 & 0 & 0 & 0 \\
0 & 0 & 0 & 0
\end{array}\right]
\end{aligned}
$$

Equation (6) hereby providing us a matrix with diagonal components as $\operatorname{diag}\left(\omega^{+} \times \omega^{-}, \fallingdotseq \times \doteqdot, 0,0\right)$ which is same as the matrix $\mathcal{F}^{\mu \nu}=\left(\begin{array}{cc}\omega^{ \pm} & 0 \\ 0 & \omega^{+}\end{array}\right)$which is taking only the diagonal elements without the $0^{\prime}$ s in diagonals and contemplating $\left(\omega^{+} \times \omega^{-} \equiv \omega^{ \pm}\right)$which shows $\mathcal{F}^{\mu \nu}$ contains only the diagonal elements which is either strong and weak or only strong, thereby eliminating the weak ones out of the league in due times.

Returning back to the old operators, if $\omega^{-}$is $\vartheta$ and $\omega^{+}$is $\vartheta+d \vartheta$ then, $\omega^{ \pm}=\vartheta^{2}+d \vartheta^{2}$ providing $\operatorname{Span} \vartheta=d \vartheta / d t$ or a function of time, we can get a mixing of the two Tensors, $J_{\text {non-null }}^{M N}$ and $J_{\text {null }}^{P Q}$ as $J^{A B} J^{C D}=\mathcal{F}^{\mu v}$ where two disease vectors $\mathcal{L}$ and $\ell$ merged to give a new vector $\mho$ yielding the form,

$$
\mho=|\mathcal{L}||\ell| \cos \theta \quad \text { where } \theta=\arccos (\mathcal{L} \cdot \ell /|\mathcal{L}||\ell|) \quad \theta \text { denoting angle of spanning of diseases }
$$

Hereby, it is plausible to conclude from equation (7) and the above paragraph, that, $\vartheta \approx \theta$ and if this happens then, the modified equation looks something like this,

$$
\operatorname{Span}=(d(\arccos (\mathcal{L} \cdot \ell /|\mathcal{L}||\ell|)) / d t)
$$

This development would take place in equation (3) as,

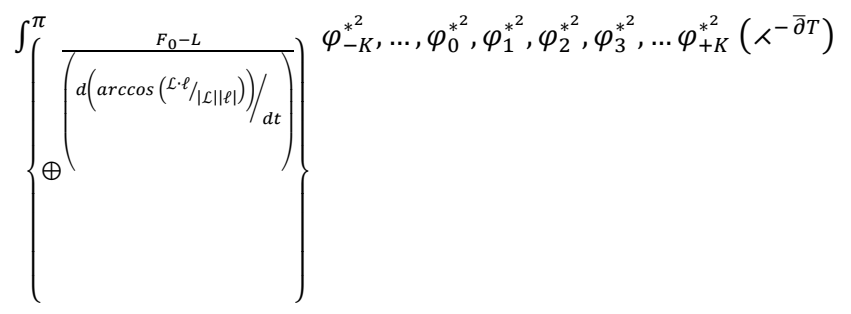

The vectors that are spanning could be defined by terms of Dual and Anti - Dual provided the vectors $\mathcal{L}$ and $\ell$ satisfies an area given by $\mathcal{L} \wedge \ell$, then its obvious that the Dual and Anti-Dual will also suppose the same wedge relation provided the Dual is $\langle\dot{\mathcal{L}} \dot{\ell}\rangle$ and Anti-Dual is $[\tilde{\mathcal{L}} \tilde{\ell}]$ satisfying a relation $\gamma\langle\dot{\mathcal{L}} \dot{\ell}\rangle \cdot \gamma^{-1}[\tilde{\mathcal{L}} \tilde{\ell}]=0$ or $\gamma^{-1}\langle\dot{\mathcal{L}} \dot{\ell}\rangle \cdot \gamma[\tilde{\mathcal{L}} \tilde{\ell}]=0$, where $\gamma, \gamma^{-1}$ is the proximity index and to satisfy this relation, the equation established given as,

$$
\gamma \text { and } \gamma^{-1} \text { can be }(\mathcal{L} \ell) \text { or } 1 /(\mathcal{L} \ell) \text { taking the value as } 0
$$

There has been a logical reason behind the above conclusion, that if the 2 Factors are there as Dual and Anti - Dual then, if the wedge of them, $\tilde{\mathcal{L}} \wedge \tilde{\ell}$ and $\dot{\mathcal{L}} \wedge \dot{\ell}$ suffices 2 different regions then one region $\alpha=\frac{\langle\dot{\mathcal{L}} \dot{\ell}\rangle}{(\mathcal{L} \ell)}$, where $(\mathcal{L} \ell)=0$ stating infinity and the region $\beta=\frac{(\mathcal{L} \ell)}{[\tilde{\mathcal{L}} \tilde{\ell}]}$, where $(\mathcal{L} \ell)=0$ stating zero or $\alpha^{-1}=\frac{(\mathcal{L} \ell)}{\langle\dot{\mathcal{L}} \dot{\ell}\rangle}$, where $(\mathcal{L} \ell)=0$ stating zero and the region $\beta^{-1}=\frac{[\tilde{\mathcal{L}} \tilde{\ell}]}{(\mathcal{L} \ell)}$, where $(\mathcal{L} \ell)=0$ stating infinity, the equation always tends to 0 . From this 2 set of equation can be constructed with extreme infection proximity magnitude denoted by infinity and low infection proximity magnitude denoted by zero given as, 


$$
\left(\frac{\langle\dot{\mathcal{L}} \dot{\ell}\rangle}{(\mathcal{L} \ell)}\left\|_{(\mathcal{L} \ell=0} \times \frac{(\mathcal{L} \ell)}{[\tilde{\mathcal{L}} \tilde{\ell}]}\right\|_{(\mathcal{L} \ell)=0}\right)_{\alpha \times \beta} \quad\left(\frac{(\mathcal{L} \ell)}{\langle\dot{\mathcal{L}} \hat{\ell}\rangle}\left\|_{(\mathcal{L} \ell)=0} \times \frac{[\tilde{\mathcal{L}} \tilde{\ell}]}{(\mathcal{L} \ell)}\right\|_{(\mathcal{L} \ell)=0}\right)_{\alpha^{-1} \times \beta^{-1}} \quad \forall(\mathcal{L} \ell)=0
$$

Spanning area could be defined by the wedge product which acts as a bivector as to the sides of a parallelogram, if it is considered and the spanning angle $\theta \sim \vartheta$ is of 2 degrees $(0,1)$ and $(1,0) \exists(1,0) \gg(0,1)$. Thereby, the spanning angle takes 2 parameters as $\theta_{0,1}$ and $\theta_{1,0} \exists \theta_{1,0} \gg \theta_{0,1}$ and based on the assumption, if the spanning area $\langle\dot{\mathcal{L}} \wedge \dot{\ell}\rangle$ is of $\theta_{0,1}$ and if it evolves through time $\Delta t$ to take a larger area $[\tilde{\mathcal{L}} \wedge \tilde{\ell}]$ is of $\theta_{1,0}$ then, there exists a population dependency factor which could be considered as a density gradient $\nabla^{\rho} \Omega_{-}^{+}$with $\nabla^{\rho}$ be the gradient of the density along with $\Omega_{-}^{+}$as the population parameter with + as high population and - as the low population, the equation takes a complex form given by,

$$
\begin{aligned}
& \sigma:=\int_{\theta_{0,1}}^{\theta_{1,0}}\left\{\langle\dot{\mathcal{L}} \wedge \dot{\ell}\rangle\left(\nabla^{\rho} \Omega_{-N} \ldots \nabla^{\rho} \Omega_{-1}, \nabla^{\rho} \Omega_{0}, \nabla^{\rho} \Omega_{+1} \ldots \nabla^{\rho} \Omega_{+N}\right) \stackrel{\Delta t}{\Rightarrow}[\tilde{\mathcal{L}} \wedge \tilde{\ell}]\left(\nabla^{\rho} \Omega_{-N} \ldots \nabla^{\rho} \Omega_{-1}, \nabla^{\rho} \Omega_{0}, \nabla^{\rho} \Omega_{+1} \ldots \nabla^{\rho} \Omega_{+N}\right)\right\}\left(\nabla^{\rho} \Omega_{-}^{+}(\mathcal{L} \wedge \ell)\right) \\
& \quad \forall \pm N \in \mathbb{R}
\end{aligned}
$$

$\sigma$ in the above equation takes a crucial parameter where, it is dependent proportionally on $\nabla^{\rho} \Omega_{+}$and inverse proportionally on $\nabla^{\rho} \Omega_{-}$. However, the integrand in equation (12) is integrated over the values $\left(\nabla^{\rho} \Omega_{-}^{+}(\mathcal{L} \ell)\right.$ ) where, the population factor $\Omega_{+}$is proportional to gradient $\nabla^{\rho}$ and for factor $\Omega_{-}$is inverse proportional to $\nabla^{\rho}$ which indicates the more the population, the more the spanning angle, the more the spanning area, the more the gradient slope but the less the time and the more peoples are inclined to infection whereas, the less the population, the less the spanning angle, the less the spanning area, the less the gradient slope and the less peoples are inclined to infection but the more the time giving us the 2 set of equations as such,

$$
\begin{aligned}
& \nabla^{\rho} \propto\left\{\Omega_{+} \propto \theta_{1,0} \propto \text { more }(\langle\dot{\mathcal{L}} \wedge \dot{\ell}\rangle \text { or }[\tilde{\mathcal{L}} \wedge \tilde{\ell}])\right\} \frac{1}{\propto} \Delta t \ldots \ldots \ldots \text { Calling this } g \\
& \nabla^{\rho} \propto\left\{\Omega_{-} \propto \theta_{0,1} \propto \text { less }(\langle\dot{\mathcal{L}} \wedge \dot{\ell}\rangle \text { or }[\tilde{\mathcal{L}} \wedge \tilde{\ell}])\right\} \propto \Delta t \ldots \ldots \ldots \text { Calling this } \mathfrak{H} \\
& g-\mathfrak{H}=\Omega_{-}^{+} \quad \exists \Omega_{-}^{+} \in \mathbb{R}
\end{aligned}
$$

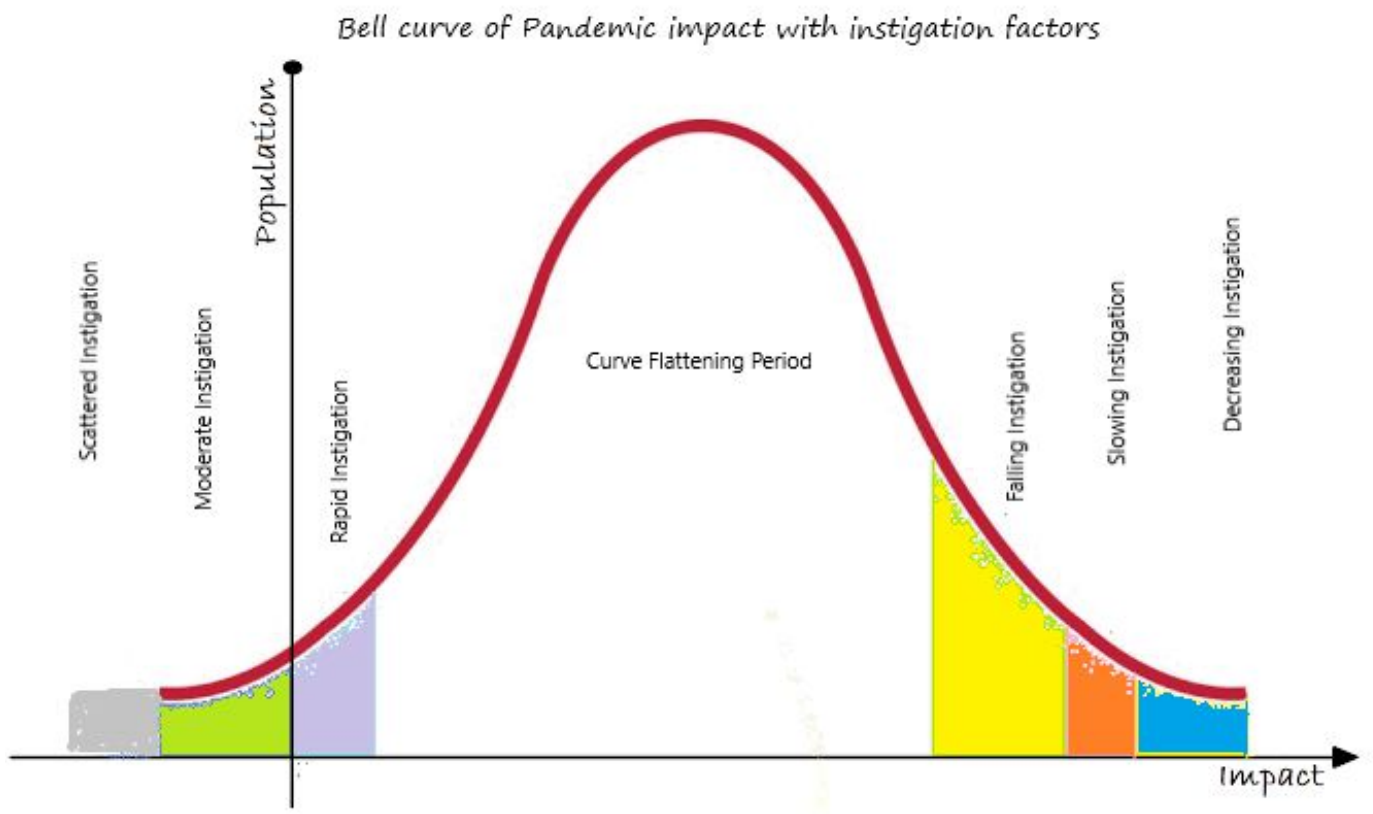

The non-trivial part of the Pandemic era is the immunization, vaccination and complete cure. These factors can be categorized by $6+1$ sub-elements as 
- Scattered Instigation which is the beginning of the spreading of the disease. Everything is in a state of chaos as, people haven't yet figured out anything about the disease. Persons are infected with no restrictions of inter-country travelling, however, the mutation strain is weak and there is no scope of vaccination.

- Moderate Instigation which is the next level (level 2) of spreading in which people are becoming aware and less chaos has been created, however, there is still inter-country travel and mutation strain is weak with no scope of vaccination. Infection increasing rate increases.

- Rapid Instigation which is the phase of the exponential increase of a disease with more and more persons getting infected, a lot of research started about the disease and people are aware of the precautions that needs to be taken, but mutation strain is getting stronger and breaking into several chains with the research actively going on for vaccination. Internal immunity against the disease has not yet been developed. Inter-country travel, inter-state travel has been restricted and lockdown phase started with the people's economic condition going down as economy has started to collapse. More death is observed in this period.

- Curve flattening Period which is considered when the active case almost becomes negligible or running in negative, as the infection rate subsides compared to the recovery rate. However, the death rate is still counting in high numbers, and human trial of the vaccination has been carried out. Economy is getting stable and restrictions on inter-country, inter-state travel are weaving out slowly by slowly.

- Falling instigation is the phase of mass infection but the recovery rate is very high as the internal antibody has been developed against the disease with a view of the finalization of vaccine trial. Death count is significantly lower.

- Slowing Instigation is the phase (or level 6) which has been identified with almost few death counts, very low infection rate, vaccination phase started and people are developing anti-bodies which are more stronger and immune to the pandemic. Recovery rate is so high that almost every person is recovering from the disease but cure is still a farfetched solution.

- Decreasing Instigation is the phase accounted for the successful response of the vaccine, death rate is almost zero except some high co-morbidity cases. Cure is on its way but its still not yet developed and this is the last phase of the disease.

Equation (15) shows us a glimpse of the result of the difference in numbers as $\mathscr{g}-\mathfrak{H}=\Omega_{-}^{+}$, however, when we try to combine it with instigation index $I$, we will get a form called $I \Omega_{-}^{+}$which can be either positive of negative supposing, $I \Omega^{+}, I \Omega^{-} \in I \Omega_{-}^{+}$. The instigation index is denoted by $6+1$ sub-parameters or elements, which has 3 degrees of freedom i.e., $(-3, \pm 1,+3)$ where -3 is the scattered instigation, moderate instigation and rapid instigation while +3 is the falling instigation, slowing instigation and decreasing instigation. The leftover factor \pm 1 is the curve flattening period. Now, denoting the instigation index with population parameter with respect to 3 Factors $(-3, \pm 1,+3)$ as $V(x)_{J,-1}=-1,-3=I \Omega^{-}, V(y)= \pm 1$ and $V(z)_{K,+1}=+1,+3=I \Omega^{+}, \exists J, K \in \mathbb{R}$, the equation can be given by,

$$
\left(V(y) \prod_{J=-1}^{-3} \prod_{K=+1}^{+3} V(x)_{J,-1}, V(z)_{K,+1}\right)
$$

Here, we can see 2 Branch Points as $-3,-1$ and $+1,+3$, these can be grouped as $(-3,-1),(+1,+3)$. Let, $\mathcal{R}$ be a set containing elements as $( \pm 1)$, the Span factor has always been $\theta$ and the immunization, vaccination and cure factor $i$ can be related via a complex form as,

$$
\mathrm{Z}=\mathcal{R} e^{i \theta} \quad \mathcal{R}, \theta \in \mathbb{R}
$$

Implying the Branch cut equations and taking natural logarithm on both sides, the following equation can be obtained,

$$
\operatorname{In} Z=\operatorname{In} \mathcal{R}+i \theta \quad \mathcal{R}, \theta \in \mathbb{R}
$$

Now, its almost time to decide that when equation (15) would give positive values and when negative values, as it can be seen from equation (16) that $V(y)$ is a constant parameter, however for $V(x)_{J}$ equation (15) gives the value as $\Omega^{+}$denoting more number of peoples infected as in equation (12) and (13) while $\Omega^{-}$as the less number of people infected $V(z)_{K}$ as in equation (12) and (14). This implies the relation as,

$$
\text { In } \mathcal{R} \propto V(y) \quad i \theta \propto V(z)_{K,+1}-V(x)_{J,-1} \quad \text { This makes the spanning angle } \theta=0
$$

Equation (19) describes the fact that, when the curve flattening period is constant and touches both the $(-3,-1)$ and $(+3,+1)$ with $\pm 1 \subseteq\{(-3,-1),(+3,+1)\}$ where, the real factor $\operatorname{In} \mathcal{R}= \pm 1$ and the imaginary factor $(-3,-1),(+3,+1)$ is $i \theta$ with $V(x)_{J-1}$ as negative and $V(z)_{K,+1}$ as positive, with $i \theta$ be positive implying the rate of infection is in a control mode when $V(z)_{K,+1}>V(x)_{J,-1}$ providing 
the difference $V(z)_{K,+1}-V(x)_{J,-1}$ is always 0 as in this case its $(-3 \times-1)-(+3 \times+1)=0$ which implies that in the control mode, the equation becomes,

$$
\text { In } Z=\operatorname{In} \mathcal{R} \in \mathbb{R}
$$

There is one factor that needs to be considered as an essential part of any disease model analysis. It's the capacity of the healthcare providers to provide care and treatment to the patients. This requires to modify equation (18) as $\operatorname{In} Z=\operatorname{In} \mathcal{R}+2 \pi n i$ where the $\theta$ is replaced by $2 \pi n$ such that $\mathcal{R}, \theta, n \in \mathbb{R}$. This is because of the rotation factor of the healthcare providers potential denoted by $\Gamma_{ \pm \mathrm{N}}$ where the potential circulates with a periodicity like, at the beginning of the pandemic, when the strain is weak, the population gradient density infection is low, the healthcares potential is high, but with the increase of everything, the strain gets moderate, the gradient becomes sloppy moderately, the healthcare is accustomed to the applications of the medicines or cures and preventions and the healthcares performance improves. It further improves with getting the cure of complete discovery of medications until a high SECOND WAVE of pandemic hits, returning everything to previous and the fact is that it happens and this $n$ in $2 \pi n i$ denotes the wave, the strain is taken from equation (3) as $\varphi_{+-K}^{*^{2}}$, the population gradient density is taken from the equation (12) as $\nabla^{\rho} \Omega_{+-N}$ and then computed equation (22) with the less and more danger areas as $\langle\dot{\mathcal{L}} \wedge \dot{\ell}\rangle$ and $[\tilde{\mathcal{L}} \wedge \tilde{\ell}]$ respectively. Therefore we get 2 equations, 1 for the healthcare potential within scaling angle as an integration over population gradient density and the master equation as follows,

$$
\begin{aligned}
& \left.\left.\int_{\theta_{0,1}}^{\theta_{1,0}} \Gamma_{-\mathrm{N}}, \ldots \ldots \ldots, \Gamma_{-1}, \Gamma_{0}, \Gamma_{+1}, \ldots \ldots \ldots, \Gamma_{+\mathrm{N}}\right\rangle_{\text {WAVE } 1} \Gamma_{-\mathrm{N}}, \ldots \ldots \ldots, \Gamma_{-1}, \Gamma_{0}, \Gamma_{+1}, \ldots \ldots \ldots \Gamma_{+\mathrm{N}}\right\rangle_{\text {WAVE } 2} \ldots\left(\nabla^{\rho} \Omega_{-}^{+}\right)
\end{aligned}
$$

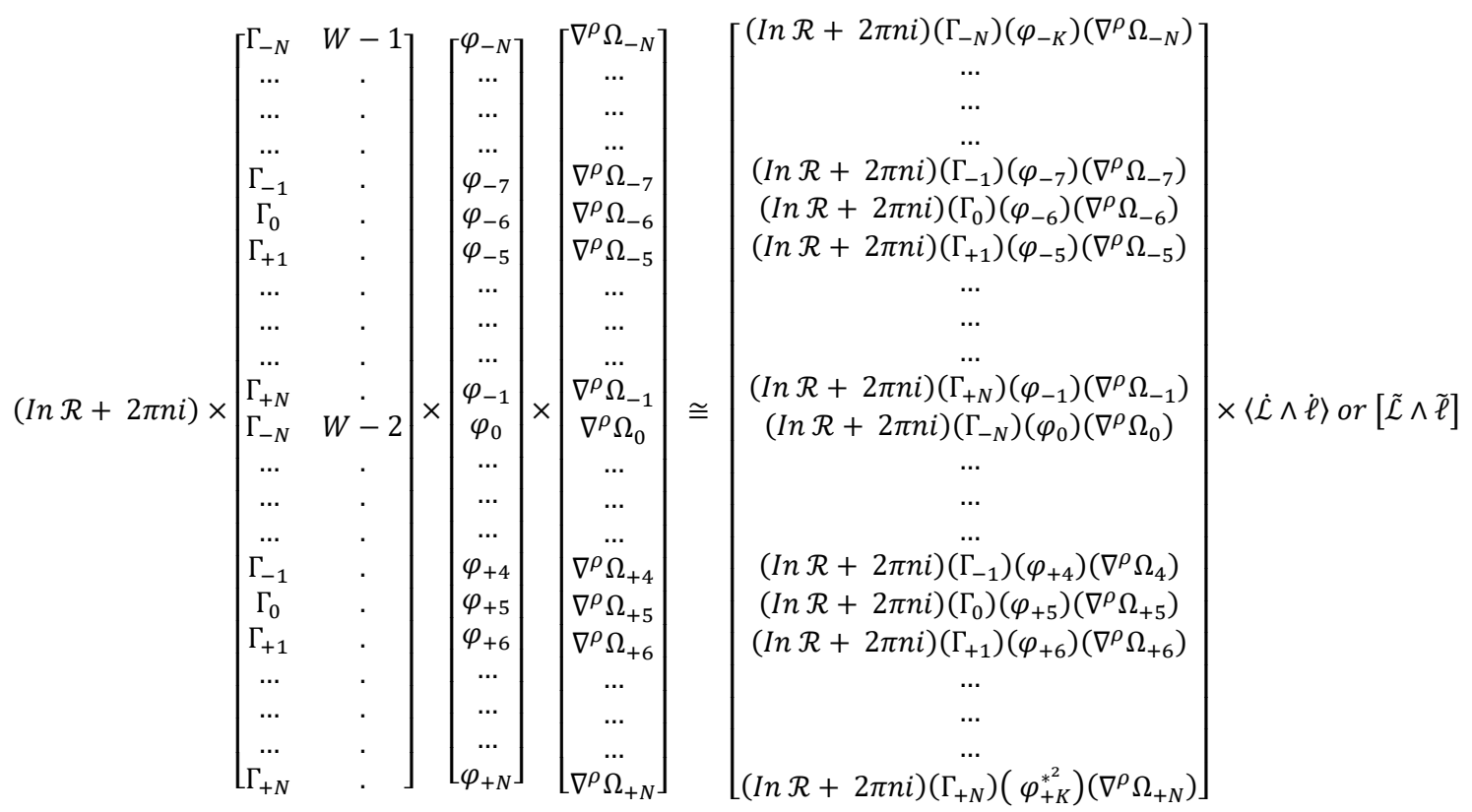

In equation (20) i.e., $\operatorname{In} Z=\operatorname{In} \mathcal{R}$ where $\mathcal{R} \in \mathbb{R} \mathcal{R}$ can take the value as +1 or -1 , if $\mathcal{R}=+1$ then $\operatorname{In} \mathcal{R}=0$ and we can neglect it, but if $\mathcal{R}=-1$ then $\operatorname{In} \mathcal{R}=1.36 i \approx 1 i$ or $i$. This indicates that when $i=+1$ which is a subset of $V(z)_{K,+1}=+1,+3=I \Omega^{+}$ showing the falling phase, the impact of Curve Flattening Period is 0 while when $i=-1$ which is a subset of $V(x)_{J,-1}=-1,-3=$ $I \Omega^{-}$the impact of Curve Flattening Period is $\sim i$, and to consider $W-1$ and $W-2$ as the 2 WAVES of the Pandemics as established in equation (22), there can be a larger number of waves like $W-3, W-4, W-5$ and so on, the factor $i$ if represents a wave cycle, such that each wave cycle is a time derivative of $\Gamma_{+-\mathrm{N}}$ as $\frac{d}{d t} \Gamma_{+-\mathrm{N}}$ then, denoting the cycle as $C$ with the index $i$ for $\langle\dot{\mathcal{L}} \wedge \dot{\ell}\rangle$ having a corresponding index $j$ for $[\tilde{\mathcal{L}} \wedge \tilde{\ell}]$ labeling each cycles as $\mathcal{C}_{a_{1} a_{2} a_{3} \ldots \ldots . . a_{n}}^{i}$ and $C_{a_{1} a_{2} a_{3} \ldots \ldots . . a_{n}}^{j}$ a Levi-Civita symbol can be established as,

$$
C_{a_{1} a_{2} a_{3} \ldots \ldots . a_{n}}^{i j}=\left\{\begin{aligned}
+1, \text { if }\left(a_{1}, a_{2}, a_{3}, \ldots \ldots ., a_{n}\right) \text { is an even permutation of }(1,2,3, \ldots \ldots, n) \\
-1, \text { if }\left(a_{1}, a_{2}, a_{3}, \ldots \ldots ., a_{n}\right) \text { is an odd permutation of }(1,2,3, \ldots \ldots, n) \\
0, \text { otherwise }
\end{aligned}\right.
$$


In $n$ cycles, where all $C_{a_{1} a_{2} a_{3} \ldots \ldots . . a_{n}}^{i}, C_{a_{1} a_{2} a_{3} \ldots \ldots . . a_{n}}^{j}$ takes the values $1,2,3, \ldots \ldots, n$, this can be represented by,

$$
\begin{aligned}
& C_{a_{1} a_{2} a_{3} \ldots \ldots a_{n}}^{i j}=\left\{\begin{array}{l}
C_{i_{1} i_{2} i_{3} \ldots \ldots . i_{n}} \\
C_{j_{1} j_{2} j_{3} \ldots \ldots . j_{n}}
\end{array}\right. \\
& C_{i_{1} i_{2} i_{3} \ldots \ldots . i_{n}} C^{j_{1} j_{2} j_{3} \ldots \ldots \ldots j_{n}}=n ! \delta_{\left[i_{1} i_{2} i_{3} \ldots \ldots \ldots i_{n}\right]}^{j_{1} j_{2} j_{3} \ldots \ldots . j_{n}}=\delta_{i_{1} \ldots \ldots \ldots \ldots i_{n}}^{j_{1} \ldots \ldots \ldots j_{n}} \\
& C_{i_{1} \ldots i_{k}, i_{k+1} \ldots \ldots . i_{n}} C^{j_{1} \ldots j_{k}, j_{k+1} \ldots \ldots . . j_{n}}=k !(n-k) ! \delta_{\left[i_{k+1} \ldots \ldots \ldots i_{n}\right]}^{j_{k+1} \ldots \ldots . j_{n}}=k ! \delta_{i_{k+1} \ldots \ldots \ldots \ldots i_{n}}^{j_{k+1} \ldots \ldots \ldots j_{n}} \\
& \mathrm{C}_{i_{1}, i_{2}, i_{3}, \ldots \ldots, i_{n}} \mathrm{C}^{i_{1}, i_{2}, i_{3}, \cdots \ldots ., j_{n}}=n ! \\
& \mathrm{C}_{j_{1}, j_{2}, j_{3}, \ldots \ldots ., j_{n}} \mathrm{C}^{j_{1}, j_{2}, j_{3}, \cdots \ldots ., j_{n}}=n !
\end{aligned}
$$

Where the factorial is marked by (!) , the generalized Kronecker delta by $\delta_{v \ldots \ldots \ldots . . .}^{\mu \ldots \ldots}$, for any $n$ the property satisfied is,

$$
\sum_{i, j, k, \ldots=1}^{n} \mathrm{C}_{i, j, k \ldots} \mathrm{C}_{i, j, k \ldots}=n !
$$

Follows the fact that,

- $\quad i$ and $j$ are Dual and Anti-Dual to $k$

- Every permutations represents odd or even

- $(+1)^{2}=(-1)^{2}=1$

- $\quad$ the permutations of $n$ element set exactly suffices to $n$ !

Therefore, if $k$ indexed permutation is $(\mathcal{L} \wedge \ell)$, then the Dual i is $\langle\dot{\mathcal{L}} \wedge \dot{\ell}\rangle$ and Anti - Dual $j$ is $[\tilde{\mathcal{L}} \wedge \tilde{\ell}]$ giving a nice relation among the 3 as expressed in equation (29).

Now, from equation (22), this relation can be properly explained as,

$$
\text { Phase Pandemic }=\left\{\begin{aligned}
+1, & \left((\operatorname{In} \mathcal{R}+2 \pi n i)\left(\Gamma_{+-N}\right)\left(\varphi^{*^{2}}{ }_{+-K}\right)\left(\nabla^{\rho} \Omega_{+-N}\right)[\tilde{\mathcal{L}} \wedge \tilde{\ell}]\right) \text { is a Anti }- \text { Dual } j \text { cycle } \\
-1, & \left((\operatorname{In} \mathcal{R}+2 \pi n i)\left(\Gamma_{+-N}\right)\left(\varphi^{*^{2}}{ }_{+-K}\right)\left(\nabla^{\rho} \Omega_{+-N}\right)\langle\dot{\mathcal{L}} \wedge \dot{\ell}\rangle\right) \text { is a Dual i cycle } \\
0, & (\mathcal{L} \wedge \ell) \|_{k}
\end{aligned}\right.
$$

Phase Pandemic will hit wave after wave if proper immunization is not developed and that immunity of affected or not effected persons is proportional to vaccination which is external immunity and internal immunity and then finally a cure. Therefore, if we take the immunity parameter as $\epsilon$ then, $\epsilon_{0}^{*}$ is the internal immunity that goes developed over time, therefore, we will take an evolution parameter for immunity as $D_{\text {Fast }}$ yielding the internal immunity as $D_{\text {Fast }} \epsilon_{0}^{*^{2}}$ having $*^{2}=1$ is the probability factor. However, there is an external (vaccination) parameter which is also dependent on time provided the strength of vaccination increases very slowly and denoted as $D_{\text {Slow }} \epsilon_{1}^{*^{2}}$ with $\epsilon_{1}^{*^{2}}$ representing external factors and is directly proportional to cure which can be denoted by $\mathbf{E}$, and from equation (30) if we take the difference between the even and odd permutations, where, we have assumed the even permutation space degree $[\tilde{\mathcal{L}} \wedge \tilde{\ell}]$ is higher than odd permutation space degree $\langle\dot{\mathcal{L}} \wedge \dot{\ell}\rangle$ as $+1>-1$ and arranging it accordingly the equation becomes,

$$
\begin{gathered}
\left((\operatorname{In} \mathcal{R}+2 \pi n i)\left(\Gamma_{+-N}\right)\left(\varphi^{*^{2}}{ }_{+-K}\right)\left(\nabla^{\rho} \Omega_{+-N}\right)[\tilde{\mathcal{L}} \wedge \tilde{\ell}]\right)-\left((\operatorname{In} \mathcal{R}+2 \pi n i)\left(\Gamma_{+-N}\right)\left(\varphi^{*^{2}}{ }_{+-K}\right)\left(\nabla^{\rho} \Omega_{+-N}\right)\langle\dot{\mathcal{L}} \wedge \dot{\ell}\rangle\right)=2 \\
\text { Or, }\left((\operatorname{In} \mathcal{R}+2 \pi n i)\left(\Gamma_{+-N}\right)\left(\varphi^{*^{2}}{ }_{+-K}\right)\left(\nabla^{\rho} \Omega_{+-N}\right)\right)([\tilde{\mathcal{L}} \wedge \tilde{\ell}]-\langle\dot{\mathcal{L}} \wedge \dot{\ell}\rangle)=2 \\
\operatorname{Or},([\tilde{\mathcal{L}} \wedge \tilde{\ell}]-\langle\dot{\mathcal{L}} \wedge \dot{\ell}\rangle)=\frac{2}{(\operatorname{In} \mathcal{R}+2 \pi n i)\left(\Gamma_{+-N}\right)\left(\varphi^{* 2}{ }_{+-K}\right)\left(\nabla^{\rho} \Omega_{+-N}\right)}
\end{gathered}
$$

If we take, the immunity factor difference, then, the difference is exactly equal to the cure factor $\mathrm{C}$ and that can be expressed as, 


$$
D_{\text {Slow }} \epsilon_{1}^{*^{2}}-D_{\text {Fast }} \epsilon_{0}^{*^{2}}=\mathrm{e}
$$

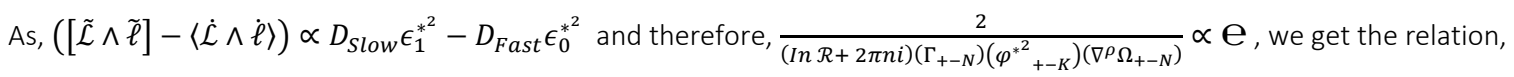

$$
\frac{2}{(\operatorname{In} \mathcal{R}+2 \pi n i)\left(\Gamma_{+-N}\right)\left(\varphi^{* 2}+-K\right)\left(\nabla^{\rho} \Omega_{+-N}\right)}=\zeta * \mathrm{e}
$$

Where $\zeta$ is the degree of the cure or how, the cure is working on the people, the cure parameter $\mathbf{e}$ can be expressed as,

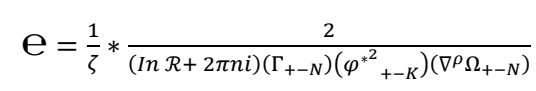

This, we get an equivalence between equation (30) and equation (36) that is between Phase Pandemic and degree of cure parameters. There is a logical reasoning behind taking the proportionality factor and summon to equation (35), the factor being, $\langle\dot{\mathcal{L}} \wedge \dot{\ell}\rangle$ is the space of low infection where the internal immunity $D_{F a s t} \epsilon_{0}^{*^{2}}$ is more, and, hence $\langle\dot{\mathcal{L}} \wedge \dot{\ell}\rangle$ is proportional to $D_{F a s t} \epsilon_{0}^{*^{2}}$. On the other hand, $[\tilde{\mathcal{L}} \wedge \tilde{\ell}]$ is the region of high infection and, here there is need for cured immunity, or external immunity $D_{\text {Slow }} \epsilon_{1}^{*^{2}}$ which is to say, $[\tilde{\mathcal{L}} \wedge \tilde{\ell}]$ is proportional to $D_{\text {Slow }} \epsilon_{1}^{*^{2}}$ and, to avoid negativity, we should subtract $[\tilde{\mathcal{L}} \wedge \tilde{\ell}]-\langle\dot{\mathcal{L}} \wedge \dot{\ell}\rangle$ and hence, this should be proportional to $D_{\text {Slow }} \epsilon_{1}^{*^{2}}-D_{\text {Fast }} \epsilon_{0}^{*^{2}}$ thereby giving the relation equation (35).

Co-morbidity $(x)$ is an important evil that comes in way of any infection and affects badly to the co-morbid patients, this also increases the death count due to severe viral attacks, however, co-morbidity can be classified as acute, moderate and deadly(or severe) based on the prevalent disease parameters like hypertension, diabetes, malignancy, immune suppressive doses for organ transplant diseases etcetera., however, not all co-morbid patients get affected badly, and the degree of affected depends upon the degree of cure parameters $\zeta$ from equation (36) which effects the cure factor $\mathbf{C}$. Classification of co-morbidity can be done as,

$$
\text { Co-morbidity }=\left\{\begin{array}{c}
-x, \text { severe } \\
+x, \text { acuute } \\
+-x, \text { moderate }
\end{array}\right\} \stackrel{\text { dependable on degree of cure parameter }}{\longrightarrow}\left\{\begin{array}{c}
\zeta^{1} \\
\zeta^{0} \\
\zeta^{-1}
\end{array}\right.
$$

Analyzing equation (37), it is evident that equation (36) could be divided into 3 sub-groups based on co-morbid populations or patients to say and the mapping as,

$$
\mathrm{e}=\left\{\begin{array}{c}
\frac{1}{\zeta^{1}} * \frac{2}{(\operatorname{In} \mathcal{R}+2 \pi n i)\left(\Gamma_{+-N}\right)\left(\varphi^{* 2}{ }_{+-K}\right)\left(\nabla^{\rho} \Omega_{+-N}\right)} \\
\frac{1}{\zeta^{0}} * \frac{2}{(\operatorname{In} \mathcal{R}+2 \pi n i)\left(\Gamma_{+-N}\right)\left(\varphi^{*^{2}}{ }_{+-K}\right)\left(\nabla^{\rho} \Omega_{+-N}\right)} \\
\frac{1}{\zeta^{-1}} * \frac{2}{(\operatorname{In} \mathcal{R}+2 \pi n i)\left(\Gamma_{+-N}\right)\left(\varphi^{*^{2}}{ }_{+-K}\right)\left(\nabla^{\rho} \Omega_{+-N}\right)}
\end{array}\right\} \stackrel{\text { dependable on co-morbidity parameter }}{\longrightarrow}\left\{\begin{array}{c}
\text { severe } \\
\text { acuute } \\
\text { moderate }
\end{array}\right.
$$

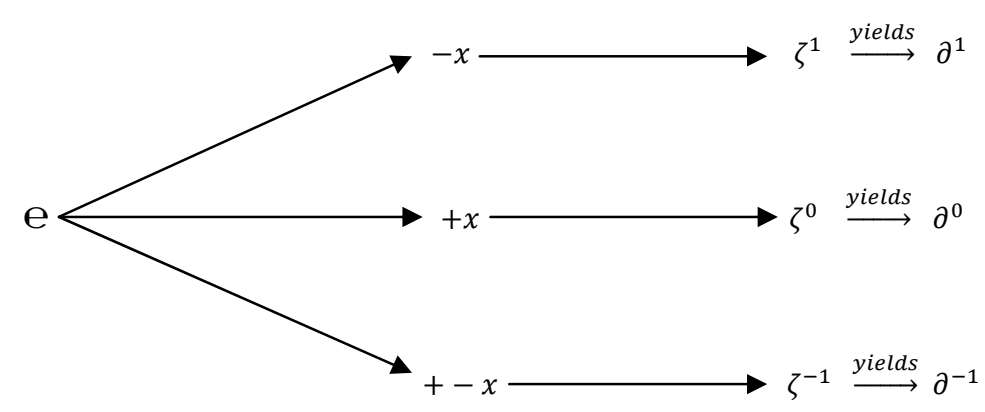

Here $\partial$ is the death mapping of co-morbid patients provided they somehow satisfies the relation as $\sim \partial^{0} \ll \partial^{-1} \ll \partial^{1}$. 
Conclusion: A specific solution is always non-trivial for any paper rather than a cumulative set of solutions. This can be used to direct the paper in one way without any divergences. The same approach has been assigned for this paper. Starting from the span factor in equation (1) to the viral factor in equation (2) to the strength of strain factor in equation (3), a body has been build up to cope with the Dual and Anti-Dual factors thereby placing the population density gradient and the $(+3,+-1,-3)$ instigation factors, which ultimately leads to the parameterization of equation (21) and the giant matrix equation (22), to account for the detailed provisions for the norms associated with the disease, which helps in concluding the vaccination, immunization, depending on the permutation factors (odd or even) as the scope to cope with the co-morbid patients encountering new diseases in the pandemic with a collective mapping for increased death for this type of patients, provides a sustainable conclusion as to encounter and restrict the pandemics from a single way developed from a bunch of parameters. This is a all together new approach than previously existing SIR models, and we hope Epidemiologists would find this model useful for real world scenario to control and check the diseases by altering the parameters of this paper.

Acknowledgement: We are grateful to Dr. Ande Murali Varaprasad (Ex-DRDO Scientist and Director of Centre for ISRO GNSS Studies at SACET) for his constant support and motivation to help young researchers like us, to publish our papers.

Declaration of Interest: The authors of this paper declared that they do not have any competing interests as related to this paper.

\section{References:}

[1] Kermack, W., \& McKendrick, A. (1927, August 01). A contribution to the mathematical theory of epidemics. Retrieved October 20, 2020, from https://royalsocietypublishing.org/doi/10.1098/rspa.1927.0118

[2] Krylova, O., \& Earn, D. J. (2013). Effects of the infectious period distribution on predicted transitions in childhood disease dynamics. Journal of The Royal Society Interface, 10(84), 20130098. doi:10.1098/rsif.2013.0098

[3] Hethcote, H. W. (2000). The Mathematics of Infectious Diseases. SIAM Review, 42(4), 599-653. doi:10.1137/s0036144500371907

[4] Miller, J. C. (2012). A Note on the Derivation of Epidemic Final Sizes. Bulletin of Mathematical Biology, 74(9), $2125-2141$. doi:10.1007/s11538-012-9749-6

[5] Kermack, W. O.; McKendrick, A. G. (1927). A contribution to the mathematical theory of epidemics. Proceedings of the Royal Society of London. Series A, Containing Papers of a Mathematical and Physical Character, 115(772), $700-721$. doi:10.1098/rspa.1927.0118

[6] Anderson, R. M., \& May, R. M. (2010). Infectious diseases of humans: Dynamics and control. Oxford: Oxford Univ. Press.

[7] Hethcote, H. W. (1989). Three Basic Epidemiological Models. Applied Mathematical Ecology Biomathematics, 119-144. doi:10.1007/978-3-642-61317-3_5

[8] Bartlett, M. S. (1957). Measles Periodicity and Community Size. Journal of the Royal Statistical Society. Series A (General), 120(1), 48. doi:10.2307/2342553

[9] Miller, J. C. (2017). Mathematical models of SIR disease spread with combined non-sexual and sexual transmission routes. Infectious Disease Modelling, 2(1), 35-55. doi:10.1016/j.idm.2016.12.003

[10] Harko, T., Lobo, F. S., \& Mak, M. (2014). Exact analytical solutions of the Susceptible-Infected-Recovered (SIR) epidemic model and of the SIR model with equal death and birth rates. Applied Mathematics and Computation, 236, 184-194. doi:10.1016/j.amc.2014.03.030

[11] Gao, S., Teng, Z., Nieto, J. J., \& Torres, A. (2007). Analysis of an SIR Epidemic Model with Pulse Vaccination and Distributed Time Delay. Journal of Biomedicine and Biotechnology, 2007, 1-10. doi:10.1155/2007/64870

[12] Yang, W., Zhang, D., Peng, L., Zhuge, C., \& Hong, L. (2020). Rational evaluation of various epidemic models based on the COVID19 data of China. doi:10.1101/2020.03.12.20034595

[13] Croccolo, F., \& Roman, H. E. (2020). Spreading of infections on random graphs: A percolation-type model for COVID-19. Chaos, Solitons \& Fractals, 139, 110077. doi:10.1016/j.chaos.2020.110077 


\section{APPENDIX}

No equation is seemingly perfect and fine-tuned if there exists a lot of uncertain and highly variable parameters with a high probability of fluctuating values. However, an equation must be constructed to signify and portrait the real life scenario. However, perturbation exists and it is this perturbation, which results in fluctuating parameters. The reality 'if satisfies a coordinate axis' then the perturbed equation would be an asymptote satisfying an equation $y=1 / x$ and the asymptote will never reach the reality coordinates. This can be best described by this below picture.

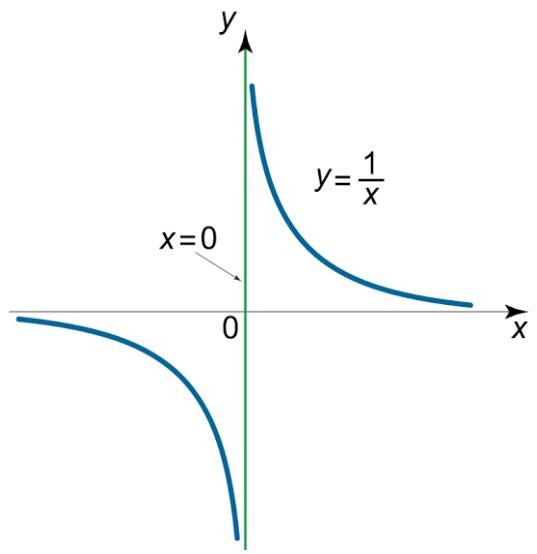

Picture courtesy:math24.net/asymptotes/

The real life affects of the 2019-2020 SARS-CoV-19 Pandemic could by analyzed by a set of splendid graphs from ourworldindata.org which uses a freely accessible CC-BY Licence and computed the chart from MIT licensed 'openware' coding platform github.com/owid/owidgrapher which we will analyze below.

Total confirmed deaths due to COVID-19 vs. Population, Oct 31, 2020 OurWorli Limited testing and challenges in the attribution of the cause of death means that the number of confirmed deaths may not be an accurate count of the true number of deaths from COVID-19.

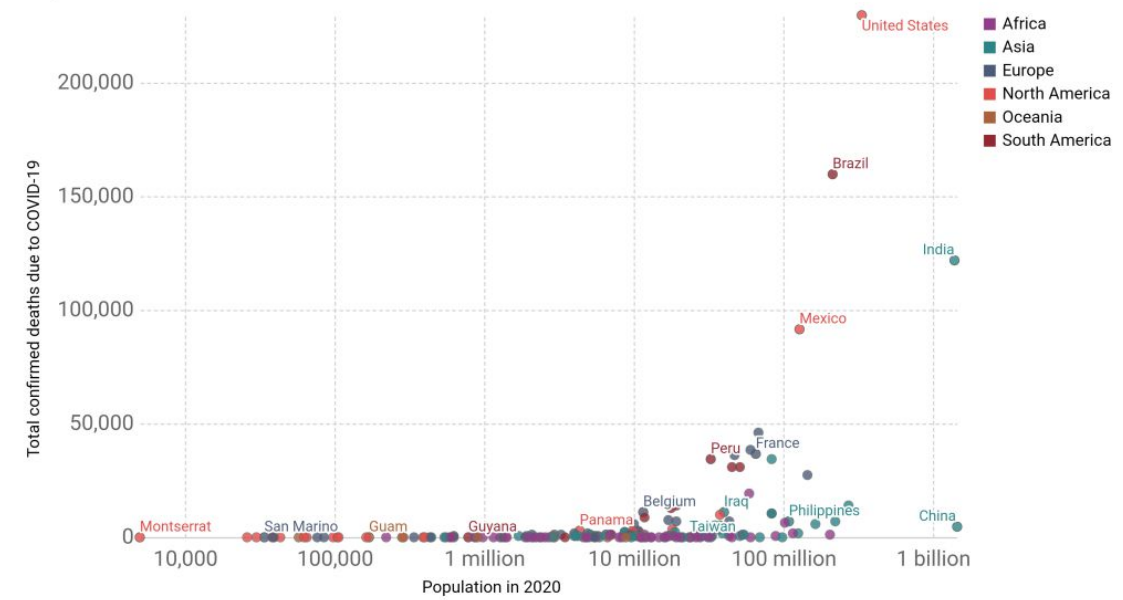

The population of India is much much more than Brazil and United States, however, due to perturbative nature of the parameters and the dependability factors as ignorance of people, crosscountry travelers, lack of restrictive measures acting on the real equation to develop this perturbative chart. 
Daily new confirmed COVID-19 deaths per million people Limited testing and challenges in the attribution of the cause of death means that the number of confirmed deaths

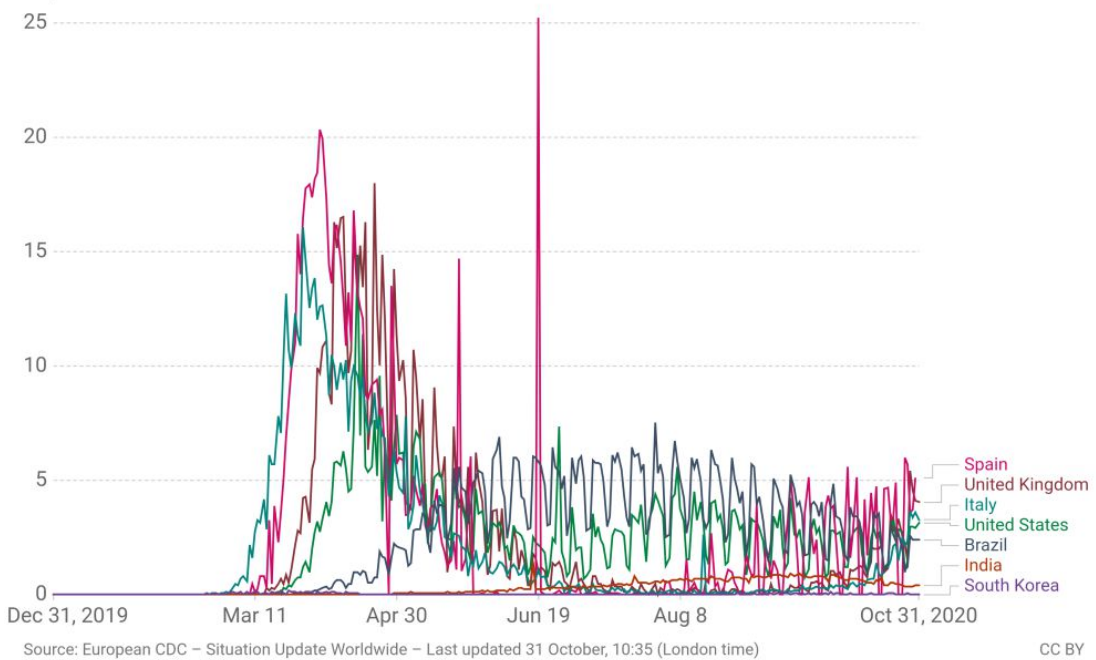

Daily new confirmed death are much higher in Spain, than compared to United States, Brazil and India, because, here the chart has been portrayed on per million people and on this note, the inspection spikes mostly in Spain which might be due to a powerful strain of virus or other unknown parameters except population density.

Daily new confirmed COVID-19 deaths per million people Shown is the rolling 7-day average. Limited testing and challenges in the attribution of the cause of death means
that the number of confirmed deaths may not be an accurate count of the true number of deaths from COVID-19.

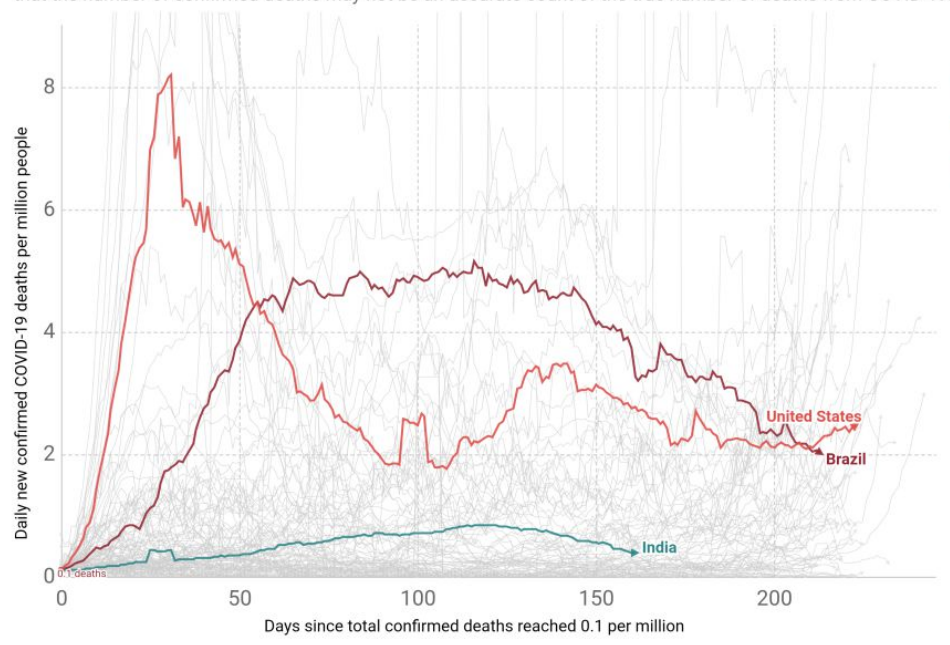

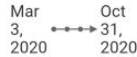

Daily new confirmed cases per million people has been concentrated on the top 3 infected countries with United States leading the group followed by Brazil and India which is directly proportional to the total and daily deaths Vs. Population. 
Total confirmed COVID-19 deaths vs. cases, Oct 31, 2020

The number of confirmed cases is lower than the number of total cases. The main reason for this is limited testing.
The grey lines show the corresponding case fatality rates, CFR (the ratio between confirmed deaths and confirmed cases).

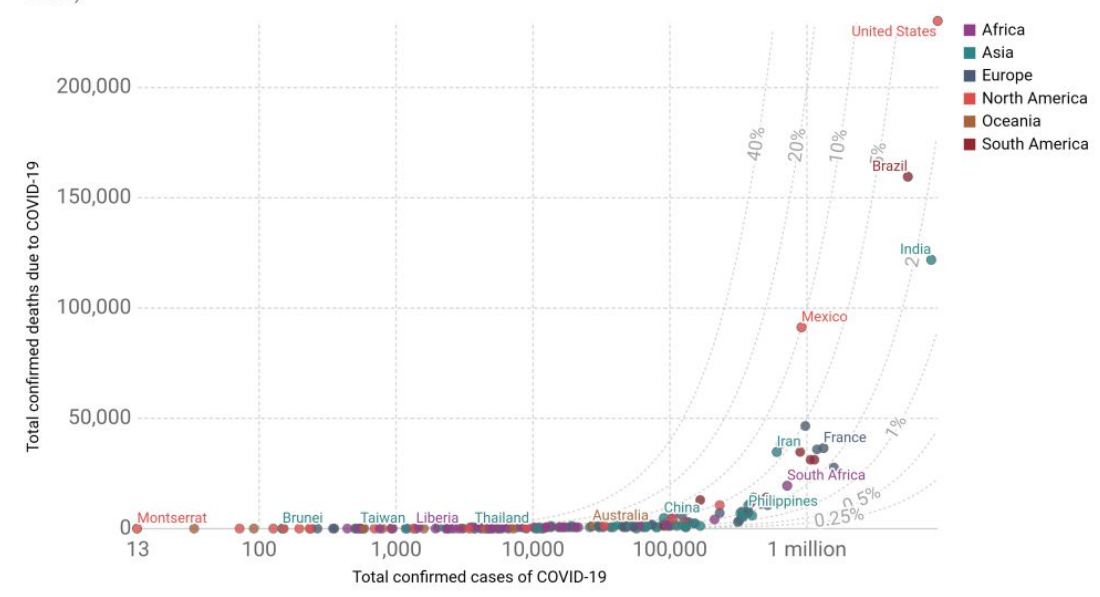

Source: European CDC - Situation Update Worldwide - Last updated 31 October, 10:35 (London time), Our World In Data OurWorldnData. org/coronavirus • CC BY
In the total Covid-19 deaths, United States as usual takes the lead followed by Brazil and India as denoted before, a proportionality relation between population wise affected and population wise deaths being greater in United States.
Total COVID-19 tests per 1,000 people

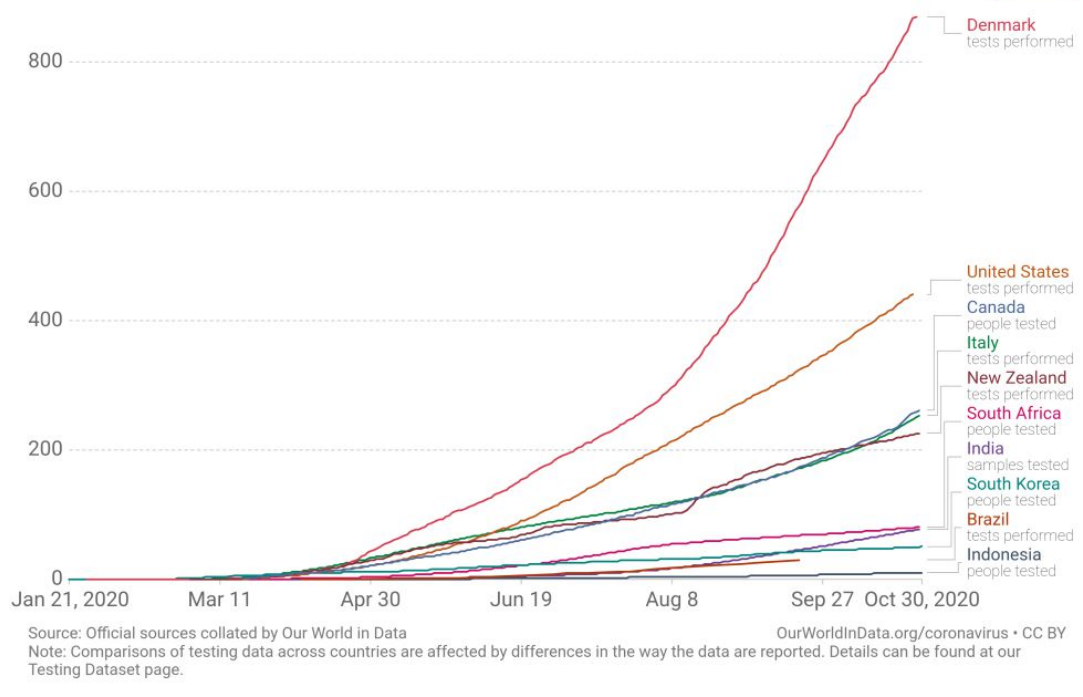

It is the perturbed affect which makes Denmark first, followed by India and Brazil with a few countries between them, although higher tests in real equation implies higher infections, but Denmark makes an absolute example of perturbations. 


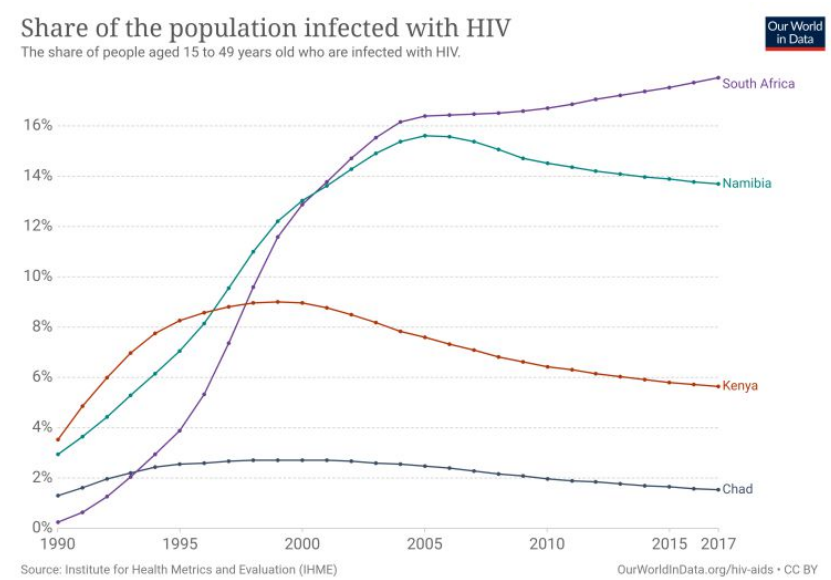

Co-morbidity with HIV is much more in South Africa.

Share of population with cancer, 1990 to 2017 Share of total population with any form of cancer, measured as the age-standardized percentage. This share has
been age-standardized assuming a constant age structure to compare prevalence between countries and through
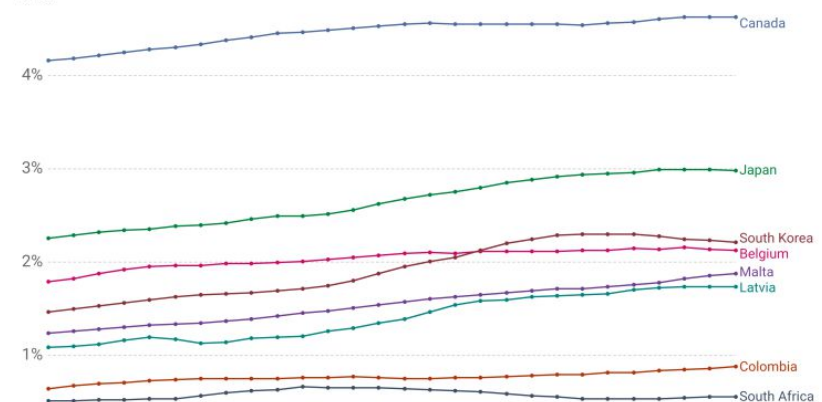

Co-morbidity with Cancer is much more in Canada.

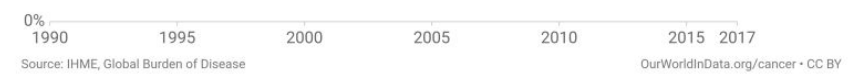

Total confirmed deaths due to COVID-19 vs. Population, Oct 31, 2020 Limited testing and challenges in the attribution of the cause of death means that the number of confirmed deaths

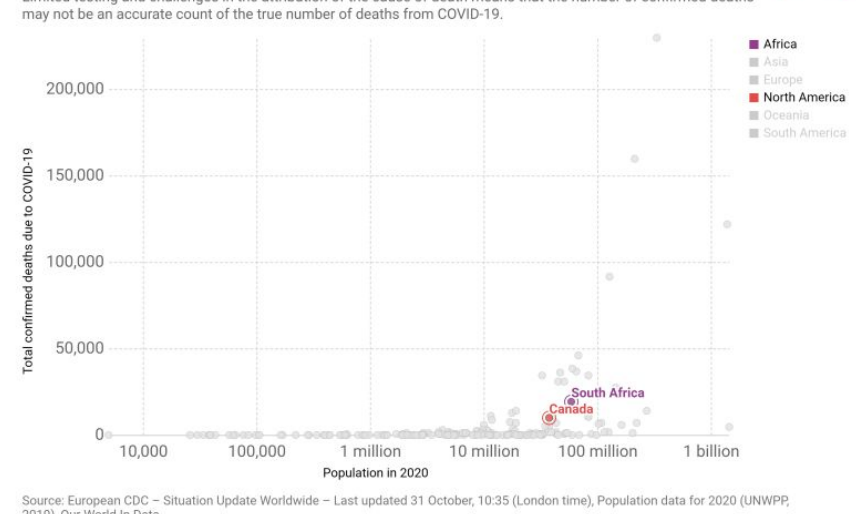

Both South Africa and Canada covers a portion of death rates due to the above mentioned Co-morbidity.

Note: All data are upto October $31^{\text {st }}$ 2020, Co-morbidity factors are much diverse, however only Cancer and HIV has been considered with relation to two countries South Africa and Canada. Data are plotted as a combination of linear and logarithmic values. 
Figures

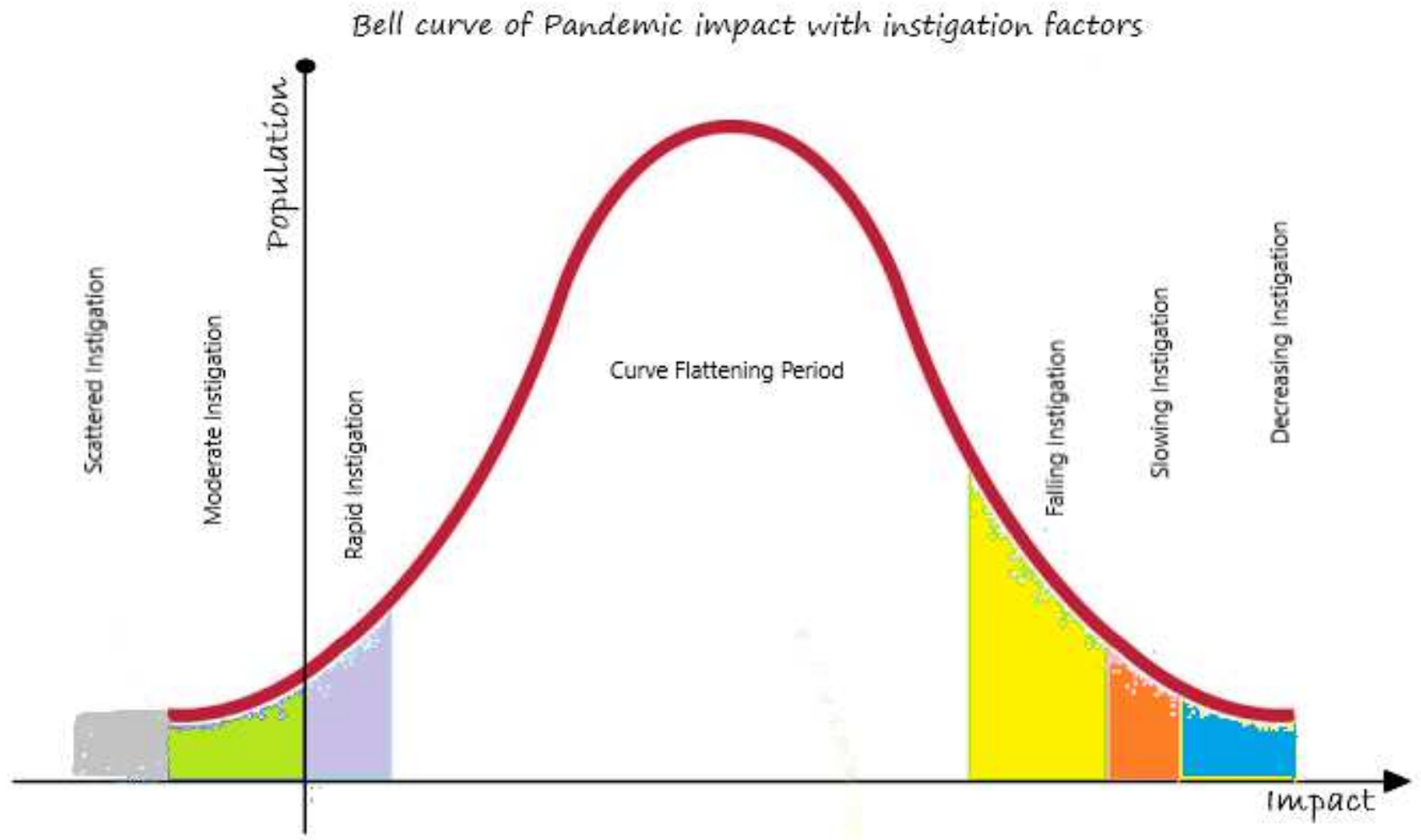

Figure 1

Bell Curve of Pandemic with Instigation Factors 